\title{
"A Characterization of a Group with Subtraction as a Binary Operation".
}

\author{
Md. Arshaduzzaman
}

\begin{abstract}
Thepresentpaperdealswith multiplicative group $A(0)$ with ' $O$ ' can be made into a subtraction group if a suitable mappingof $A(0)$ exists.Moreoverthegreatmathematician B.M. Schein[2] raised the problem of characterizi $n g$ semigroups which can become subtraction semi-groups, when a subtraction operatoris defined on them in terms of $t$ he group composition. In Bohdan Zelinka[1]solved this problem partially by showing atomic subtraction semigrou ps canbe characterized as cancellative semigroups containing ' 0 '.
\end{abstract}

Mathematics Subject Classification: $20 M 20$

Keywords and phrases : subtraction algebra, subtraction semigroup, subtraction group.

\section{Introduction}

In mathematics, a semi group is an algebraic structure consisting of a set together with an associative binary operation. A semi group generalizes a monoid in that a semigroup need not have anidentity element. It also (originally) generalized a group (a monoid with all inverses) to a type where every element did not have to have an inverse, thus the name semigroup.

The formal study of semigroups began in the early 20th century. Semigroups are important in many areas of mathematics because they are the abstract algebraic underpinning of "memoryless" systems: time-dependent systems that start from scratch at each iteration. In applied mathematics, semigroups are fundamental models for linear time-invariant systems. In partial differential equations, a semigroup is associated to any equation whose spatial evolution is independent of time. The theory of finite semigroups has been of particular importance in theoretical computer science since the 1950s because of the natural link between finite semigroups and finite automata. In probability theory, semigroupsare associated with Markov processes (Feller 1971).

RecentlyB.M. Schein[2] raised the problem of characterizing semi-groups which can become subtraction semigroups, when a subtraction operator is defined on them in terms of the group composition. In Bohdan Zelinka[1] solved this problem partially by showing atomic subtraction semigroups can be characterized as cancellative semigroups containing ' 0 '. The present paper deals with multiplicative group $A(0)$ with ' 0 ' can be made into a subtraction group if a suitable mapping of $A(0)$ exists.

\section{Some Definitions and Auxiliary Results}

This section contains some definitions and auxiliary results.

Definition 2.1. A pair $(A,-)$ where $A$ is a nonempty set and '-' is a binary operation on $A$ is called a subtraction algebra if
(a) $x-(y-x)=x$;
(b) $x-(x-y)=y-(y-x)$ and
(c) $(x-y)-z=(x-z)-y$ for all $x, y, z \in A$.

Definition 2.2. A triple $(A,-,$.$) is called a subtraction semigroup if$

(1) $(A,-)$ is a subtraction algebra;

(2) $(A, \cdot)$ is a semigroup and

(3) $x(y-z)=x y-x z$ and $(y-z) x=y x-z x$ for all $x, y, z \in A$.

Definition 2.3. If $(G,$.$) is a group with identity e$, by the group $G$ with 0 we understand the set $G \cup\{0\}$ [where ' 0 ' is an element not in $G$ ] with multiplication $x \cdot y$ as in $G$ if $x, y \in G$ and $x \cdot y=0$ if $0 \in\{x, y\}$.

we know that in every subtraction algebra $A$ there exists an element 0 such that $0=a-a$ for all $a \in A$. 
Definition 2.4. A triple $(A,-,$.$) is called a subtraction group if$

(i) $(A,-,$.$) is a subtraction semigroup and$

(ii) $A-10\}$ is a group with the multiplication inherited from $A$.

\section{Main Results Now we prove our main results.}

Theorem 3.1. Suppose $A(0)$ is a subtraction group and $A=A(0)-\{0\}$. Define

$\lambda: A(0) \rightarrow A(0)$ by $\lambda(x)=e-x$ where ' $e$ ' is the identity in $A$. Then

$$
\text { (i) } x \lambda\left(x^{-1} y \lambda\left(y^{-1} x\right)\right)=x
$$

(ii) $x \lambda^{2}\left(x^{-1} y\right)=y \lambda^{2}\left(y^{-1} x\right)$ and

(iii) $x \lambda\left(x^{-1} z\right) \lambda\left[\left(\lambda\left(x^{-1} z\right)\right)^{-1} x^{-1} y\right]=x \lambda\left(x^{-1} y\right) \lambda\left[\left(\lambda\left(x^{-1} y\right)\right)^{-1_{x}-1_{z}}\right.$ for all $x, y, z \in$

A

Proof. (i) $x \lambda\left(x^{-1} y \lambda\left(y^{-1} x\right)\right)=x \lambda\left(x^{-1} y\left(e-y^{-1} x\right)\right)$

$=x \lambda\left(x^{-1} y-e\right)=x\left[e-\left(x^{-1} y-e\right)\right]=x e=x$

(ii) $x \lambda^{2}\left(x^{-1} y\right)=x \lambda\left(e-x^{-1} y\right)$

$=x\left[e-\left(e-x^{-1} y\right]=x-x\left(e-x^{-1} y\right)=x-(x-y)\right.$.

Similarly $y \lambda^{2}\left(y^{-1} x\right)=y-(y-x)$.

By (b) of definition 1.1 we get $x \lambda^{2}\left(x^{-1} y\right)=y \lambda^{2}\left(y^{-1} x\right)$.

(iii) L.H.S $=x \lambda\left(x^{-1} z\right) \lambda\left[\left(\lambda\left(x^{-1} z\right)\right)^{-1} x-1 y\right]=x\left(e-x^{-1} z\right) \lambda\left[\left(e-x^{-1} z\right)^{-1} x-1_{y}\right]$

$=(x-z) \lambda\left[\left(x\left(e-x^{-1} z\right)\right)^{-1} y\right]=(x-z) \lambda\left[(x-z)^{-1} y\right]$

$=(x-z)\left[e-(x-z)^{-1} y\right]=(x-z)-y$.

Similarly R.H.S $=(x-y)-z$.

This proves (iii).

Theorem 3.2. Suppose $A$ is a group and $A(0)$ is the corresponding group with

0. Suppose $\lambda: A \rightarrow A$ has the properties (i),(ii) and (iii) described in The-

Orem 2.5. Then $A(0)$ is a subtraction group if we define $x-y=x \lambda\left(x^{-1} y\right)$,

$0-y=0$ and $y-0=y$ for all $x, y \in A$.

Proof. We need to prove

(a) $x-(y-x)=x$,

(b) $x-(x-y)=y-(y-x)$ and

(c) $(x-y)-z=(x-z)-y$ for all $x, y, z \in A(0)$.

Let us first check these for $x, y, z \in A$.

(a) $x-(y-x)=x-\left(y \lambda\left(y^{-1} x\right)\right)$

$=x \lambda\left(x^{-1} y \lambda\left(y^{-1} x\right)\right)=x$. [by(i)]

(b) $x-(x-y)=x-x \lambda\left(x^{-1} y\right)=x \lambda\left[x^{-1} x \lambda\left(x^{-1} y\right)\right]=x \lambda^{2}\left(x^{-1} y\right)$.

Similarly $y-(y-x)=y \lambda^{2}\left(y^{-1} x\right)$.

Therefore $x-(x-y)=y-(y-x)$. [by(ii)]

(c) $(x-y)-z=x \lambda\left(x^{-1} y\right)-z=x \lambda\left(x^{-1} y\right)\left[\left(x \lambda\left(x^{-1} y\right)\right)^{-1} z\right]=x \lambda\left(x^{-1} y\right)\left[\left(\lambda\left(x^{-1} y\right)\right)^{-1} x-1_{z}\right]$.

Similarly $(x-z)-y=x \lambda\left(x^{-1} z\right)\left[(\lambda(x-1 z))-1_{x}-1 y\right]$ and

hence $(x-y)-z=(x-z)-y$. [by (iii)]

Now if one or more elements of $x, y, z$ are equal to zero, an easy check reveals

that the conditions (a),(b) and (c) listed at the beginning of this proof are valid in this case too. 


\section{Conclusion:}

In B.M. Schein[2] raised the problem of characterizing semi-groups which can become subtraction semigroups, when a subtraction operator is defined on them in terms of the group composition. In Bohdan Zelinka[1] solved this problem partially by showing atomic subtraction semigroups can be characterized as cancellative semigroups containing ' 0 '. In this paper, we remove the restriction of atomicity and show that a multiplicative group $A(0)$ with ' 0 ' can be made into a subtraction group if a suitable mapping of $A(0)$ exists.

\section{References :}

[1] Bohdan Zelinka: Subtraction Semigroups, Mathematical Bohemica, Vol.120(1995), N0.4, 445-447.

[2] Schein B.M.: Difference semigroups, Communications in Algebra 20(1992), 2153-2169.

[3] Abbott, J.C.: Sets, Lattices and Boolean Algebras, Allyn and Bacon, Bosten, 1969.

[4] J. C. Beidleman, A note on Regular near-rings, J. Indian Math. Soc. 33 (1969), 207-210.

[5] J. R. Clay, The near-rings on groups of low order, Math. Z. 104 (1968), 364-371.

[6] K. H. Kim, On Subtraction Semigroups, Scientiae Mathematicae Japonicae 62 (2005),no. 2, $273-280$.

[7] Meldrum, Varieties and d.g. near-rings, Proc. Edinburgh Math. Soc. (series 1) 17 (1971), 271-274

[8] G. Mason, Strongly regular near-rings, Proc. Edinburgh Math. Soc. 23 (1980), 27-35.

[9] G. Pilz, Near-rings, North-Holland, Amsterdam, 1983.

[10] E. H. Roh, K. H. Kim, and Jong Geol Lee, On Prime and Semiprime ideals in Subtrac-tion Semigroups, Scientiae Mathematicae Japo nicae 61 (2005), no. 2, 259-266. 